\title{
Energy Harvesting from Vibrations of a Two-Degree-of-Freedom Mechanical System
}

\author{
J. SNAMinA* AND P. ORKISZ \\ AGH University of Science and Technology, Faculty of Mechanical Engineering and Robotics \\ Department of Process Control, Al. A. Mickiewicza 30, 30-059 Krakow, Poland
}

\begin{abstract}
In the paper, the energy harvesting from vibration of two-degree-of-freedom mechanical system is analyzed. The considered system consists of two mass linked in series by means of springs and dampers. The kinematic excitation of the system was assumed. The energy conversion system was placed in the suspension of lower mass. As a result of the analysis, the methods to increase the energy harvesting from vibration were proposed. The laboratory stand has been built and a series of measurements performed. Results of numerical simulations and measurements are presented in graphs.
\end{abstract}

DOI: 10.12693/APhysPolA.125.A-174

PACS: 84.60.-h, 46.40.-f, 45.30.+s

\section{Introduction}

Recently there has been a growing interest in energy harvesting from mechanical systems executing vibrations [1-3]. In harvesting systems, some part of mechanical energy is converted into electrical energy by means of special energy subsystems $[4,5]$ and is saved in capacitors [6] or rechargeable batteries. The energy conversion is realized by using linear DC motors or piezoelectric devices. The energy storage takes place in electrical networks containing bridge rectifiers and capacitors or batteries. Recently, a number of studies were published (cf. [7] as an example) on replacing bridge rectifiers with power-electronic switches in rectifier circuitry. In order to be able harvest as large amount of energy as possible, both processes - energy conversion and energy storage - should be optimized.

In the paper, the strategy to increase the energy harvesting from vibration of a two-degree-of-freedom (DOF) mechanical system is analyzed. Guidelines for the energy conversion and the energy storage process are formulated. The efficiency of the energy storage process is mainly associated with the speed of charging the capacitors. A special efficiency coefficient was introduced in calculations to take this into account.

The considered system consists of two mass linked in series by means of springs and dampers. Such structure is often used as a simple model of suspension systems. The kinematic excitation of the system was assumed. The energy conversion system was placed in the suspension of the lower mass.

\section{Description of the system}

Calculations and measurements were done for a system that is shown in Fig. 1. The system consists of two masses that can be moved in vertical direction. The support of the system is connected with moving part of an exciter.

\footnotetext{
* corresponding author; e-mail: snamina@agh.edu.pl
}

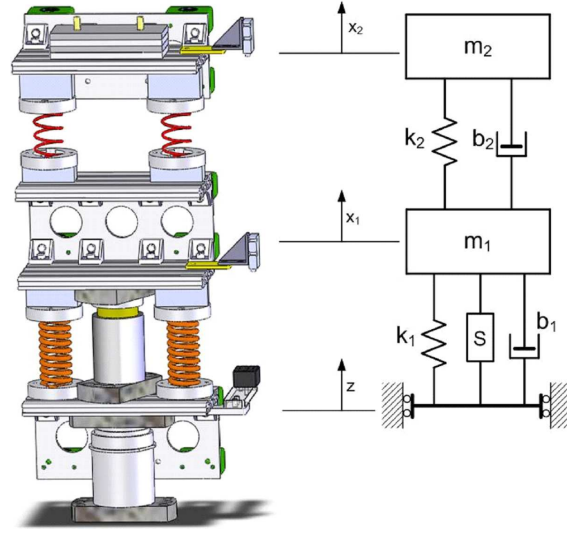

Fig. 1. Scheme of laboratory stand.

The system may be a simple model of many different objects. For example, a quarter-car model has the same structure which is very commonly used for investigating dynamics of a car suspension [8]. Similarly, vibrations of sprung transport platforms can be, to the first approximation, studied with the proposed model.

Two linear motors are used in the laboratory stand. The lower motor works as an exciter. The platform attached to the moving part of the motor moves according to the prescribed function $z(t)$. The second motor, placed in the suspension of the lower mass, converts mechanical energy into electric energy. The force exerted by the linear motor is denoted by $F(t)$ and it is applied to the lower mass and to the platform attached to the moving part of the motor.

\section{Mathematical model}

The proposed system is a simple 2-DOF mechanical system with energy harvesting subsystem consisting of a linear motor and an energy storage system. It was assumed that electric energy is accumulated in a capacitor. In order to describe the state of the system, the mechanical subsystem and electrical subsystem should be considered separately. They are shown in Fig. 2. 


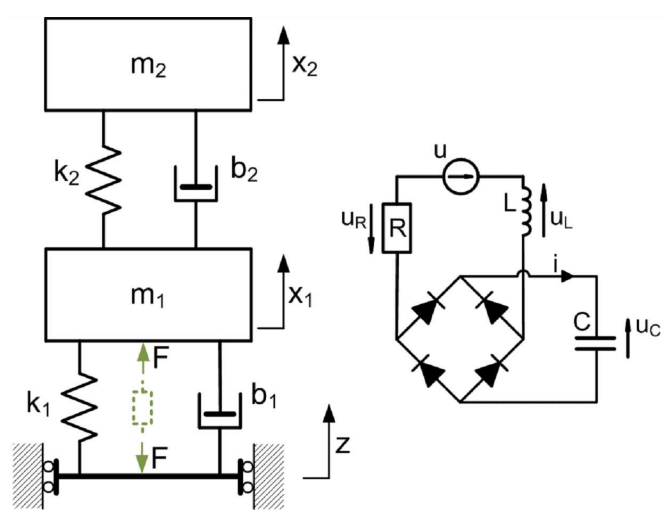

Fig. 2. Mechanical and electrical subsystem.

Equations describing the vibrations of both masses in relation to the static equilibrium position of the system take the following form:

$$
\left\{\begin{array}{c}
m_{1} \ddot{x}_{1}=k_{1}\left(z-x_{1}\right)+b_{1}\left(\dot{z}-\dot{x}_{1}\right) \\
-k_{2}\left(x_{1}-x_{2}\right)-b_{2}\left(\dot{x}_{1}-\dot{x}_{2}\right)+F \\
m_{2} \ddot{x}_{2}=k_{2}\left(x_{1}-x_{2}\right)+b_{2}\left(\dot{x}_{1}-\dot{x}_{2}\right)
\end{array}\right.
$$

where coordinates and coefficients are described in Fig. 2.

In the calculations, the simplest form of electromechanical coupling is assumed that can be realized by a permanent-magnet DC motor. In this model, the voltage $u$ is related to relative velocity $\left(\dot{z}-\dot{x}_{1}\right)$ and the force $F$ is related to current $i$ according to the following equations:

$$
\left\{\begin{array}{l}
u=\kappa_{1}\left(\dot{z}-\dot{x}_{1}\right) \\
F=\kappa_{2} i
\end{array},\right.
$$

where $\kappa_{1}, \kappa_{2}$ are the motor constants [9]. When $\kappa_{1}=$ $\kappa_{2}=\kappa$ the electric power equals mechanical power and the linear motor converts the electric energy into mechanical energy without any loss.

Equations of the electrical subsystem resulting from the Kirchhoff's voltage law and basic properties of the capacitor can be written in the form:

$$
\begin{aligned}
& C L \frac{\mathrm{d}^{2} u_{c}}{\mathrm{~d} t^{2}}+C R \frac{\mathrm{d} u_{c}}{\mathrm{~d} t}= \\
& \quad \frac{1}{2}\left(1+\operatorname{sgn}\left(|u|-u_{c}\right)\right)\left(|u|-u_{c}\right), \\
& i=C \frac{\mathrm{d} u_{c}}{\mathrm{~d} t}
\end{aligned}
$$

where $L$ is the inductivity and $R$ is the resistance of the linear motor winding, and $\mathrm{C}$ is the capacitance of the capacitor. In further considerations, the simplest diode model called the "ideal diode" and defined as a device having zero resistance when forward-biased and infinite resistance when reverse-biased will be assumed.

Equations (1)-(3) describe evolution of the considered electromechanical system.

\section{Efficiency of energy storage process}

During the process of charging a capacitor a part of electric energy is converted into heat and dispersed in the surrounding space. The dispersed energy should be as small as possible in order to retain good efficiency of the energy accumulation process. It is apparent that the speed of capacitor charging is the main parameter influencing efficiency of the energy accumulation process.

The aim of analysis is the assessment of efficiency in the simple case of charging the capacitor by current flowing trough resistor as a result of increasing voltage of the source. The circuit including voltage source, resistor, and capacitor is shown in Fig. 3. In analysis, the inductor was omitted because the voltage across it is small during slow process of charging the capacitor.

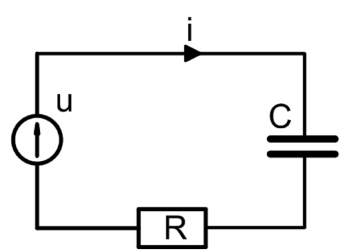

Fig. 3. Capacitor charging circuit.

In the assumed circuit, the speed of charging the capacitor is directly related with circuit time constant $T$ that is the product of resistance $R$ and capacitance $C$. The speed of charging depends also on the source voltage increase rate. In calculations, the following formula describing the source voltage was assumed:

$$
u(t)=u_{0}\left(1-e^{-\frac{t}{T_{u}}}\right),
$$

where $T_{u}$ is the time parameter and $u_{0}$ is the asymptotic voltage.

The source provides the energy for the circuit. It should be accumulated in capacitor but due to the JouleLenz effect, a part of electric energy is turned into heat in the resistor. The differential equation describing the charge $q(t)$ flowing in the circuit and accumulating in the capacitor has the following form:

$$
R C \frac{\mathrm{d} q}{\mathrm{~d} t}+q=C u(t) .
$$

Assuming that the capacitor was completely discharged at $t=0$ and taking into account the formulae (4) describing the voltage $u(t)$, the solution of Eq. (5) is

$$
q(t)=C u_{0}\left(1-\frac{1}{T_{u}-T}\left(T_{u} e^{-\frac{t}{T_{u}}}-T e^{-\frac{t}{T}}\right)\right) .
$$

The above solution describing the capacitor charge as a function of time allows calculating the energy $E_{c}(t)$ accumulated in the capacitor:

$$
E_{c}(t)=\frac{1}{2} C u_{0}^{2}\left(1-\frac{1}{\alpha-1}\left(\alpha e^{-\frac{t}{T_{u}}}-e^{-\alpha \frac{t}{T_{u}}}\right)\right)^{2},
$$

where coefficient $\alpha$ is the ratio of the source time parameter $T_{u}$ to the circuit time constant $T$.

Electric energy $E_{R}$ converted into heat in the resistor can be calculated by first determining the current as a function of time. Next, the energy converted into heat is calculated as the integral of the product of resistance and current squared. Electric energy $E_{R}$ converted into heat is finally described by the formula:

$$
E_{R}(t)=\frac{1}{2} C u_{0}^{2} \frac{1}{(\alpha-1)^{2}}\left\{\alpha\left(1-e^{-2 \frac{t}{T_{u}}}\right)\right.
$$




$$
\left.-4 \frac{\alpha}{1+\alpha}\left[1-e^{-\frac{t}{T_{u}}(1+\alpha)}\right]+1-e^{-2 \alpha \frac{t}{T_{u}}}\right\} .
$$

The energy storage efficiency coefficient $\eta$ is defined as the ratio of the energy accumulated in capacitor (useful effect) to the source energy (expended energy) that is a sum of the energy accumulated in capacitor and the energy converted into heat:

$$
\eta=\frac{E_{C}}{E_{C}+E_{R}} .
$$

Plots of the efficiency coefficient, versus dimensionless time $\tau=t / T_{u}$ are shown in Fig. 4 for various values of coefficient $\alpha$.

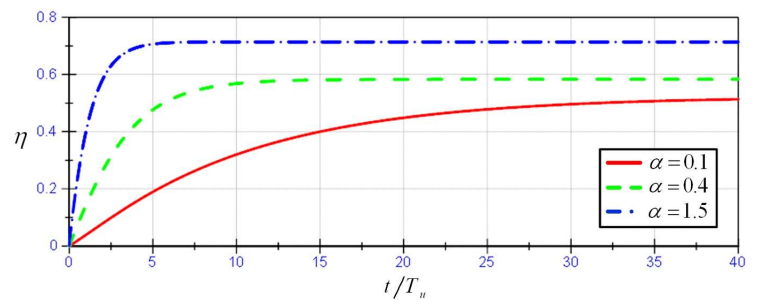

Fig. 4. Efficiency coefficient as a function of dimensionless time $\tau=\mathrm{t} / \mathrm{Tu}$.

Assuming that duration of the process of charging the capacitor approaches infinity $(t \rightarrow \infty)$, the efficiency coefficient value tends to the following limit:

$$
\eta_{\infty}=\lim _{t \rightarrow \infty} \frac{E_{c}}{E_{C}+E_{R}}=\frac{1+\alpha}{2+\alpha} .
$$

The form of the formula (10) shows that the limit value of the efficiency coefficient is a simple function of the ratio $\alpha$ of the source time parameter $T_{u}$ to the circuit time constant $T$. If $T_{u}$ approaches zero, the source voltage takes the form of a step signal. Voltage $u(t)$ reaches its maximum value $u_{0}$ extremely fast. In this case, the efficiency is equal to 0.5 and this is the minimum possible value of this quantity. If the voltage $u(t)$ reaches its maximum value $u_{0}$ slowly, the efficiency is larger. This regularity is true independently of the circuit time constant $T$. The plot of the efficiency coefficient as a function of ratio of the source time parameter $T_{u}$ to the circuit time constant $T$ is shown in Fig. 5.

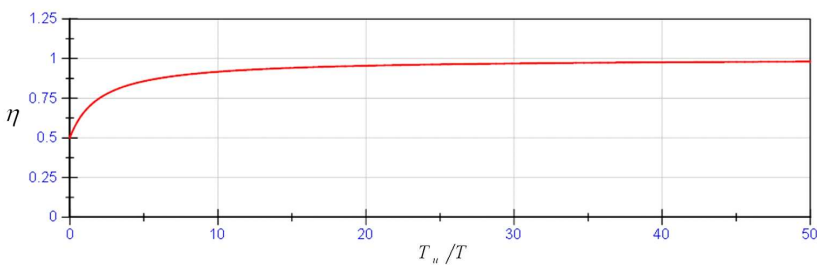

Fig. 5. Efficiency coefficient as a function of ratio $\alpha$.

Results of the presented calculations can be used as a base for selection of parameters of the energy accumulation subsystem. The energy accumulation process will be effective when the source time parameter $T_{u}$, associated with the source voltage increase rate, is sufficiently larger than the circuit time constant $T$. If the voltage source is associated with vibrating system, the voltage increase rate is directly associated with frequency of vibrations. Large values of $T_{u}$ are associated with longer times of the charging process. It is apparent that there is a conflict between the efficiency and the charging rate. The energy storage process efficiency coefficient $\eta$ is reduced additionally as a consequence of increasing capacitance, because in this case the circuit time constant increases. Finally, we come to conclusion that the storage system with large capacitance and large charging rate has in general a small energy storage efficiency.

\section{Laboratory workstation}

The laboratory workstation is presented in Fig. 6 . The workstation structure is supported by frame (1), to which components of the vibration reduction system are mounted. Three moveable platforms are connected to vertical slides by means of linear bearings. Platforms are linked to each other by means of springs. The lower platform is linked with moving part of the LA30 linear electro-dynamic motor (10) and with the magnetostrictive displacement transmitter (11). Between the lower platform and the intermediate platform, the LA25 linear motor (8) is placed as a part of the energy harvesting system. The workstation is additionally provided with incremental heads, which are linear encoders (2) and (6). The displacement transmitters were selected in order to achieve a high measuring resolution of $0.01 \mathrm{~mm}$ for platforms (3) and (5), and $0.1 \mathrm{~mm}$ for platform (9).

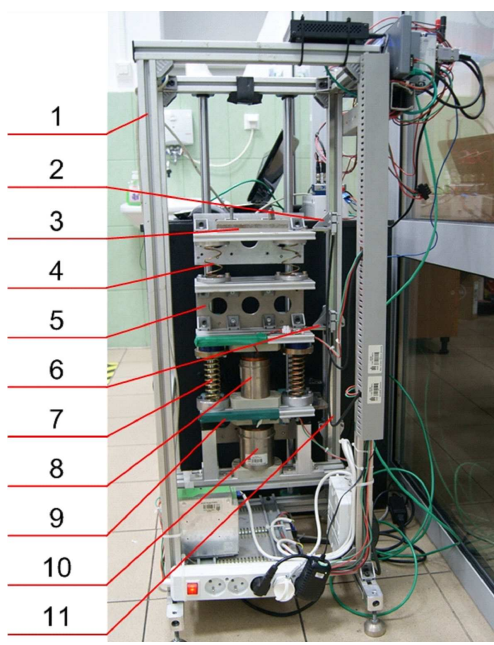

Fig. 6. Laboratory workstation.

The control and measuring system was divided into two parts, the first part for controlling the exciter and the second part for measuring test signals. The proposed structure of the control and measuring system is shown in Fig. 7. The tests were carried out using the LabView programming environment. Control algorithms were executed at the hardware level. The control section consisted of cRIO controller provided with a pro- 


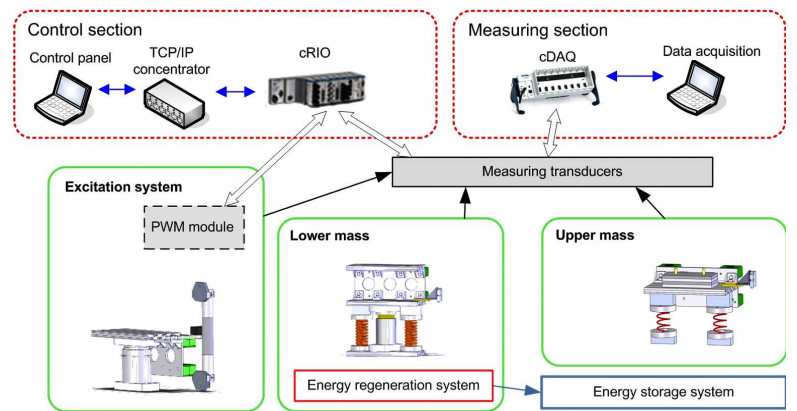

Fig. 7. Structure of the control and measuring system.

grammable array of logic gates FPGA, real time processor, input/output modules, and the TCP/IP communication module. There were two ways of archiving the control data - by exchanging data streams with the control panel and sharing prepared files with the FTP service.

The measuring section comprised a cDAQ controller with analog input measuring cards. It allowed for scaling and recording the measurement data on $\mathrm{PC}$ computer. The measuring converters made possible to record displacement of platforms, as well as the current and the voltage in the energy harvesting system.

The block diagram of the proposed energy harvesting system is shown in Fig. 8. The system is divided into two subsystems. The first is the energy regeneration subsystem and the second is the energy storage subsystem. Taking into account the oscillatory character of the motion of the mechanical system, the full-wave rectifier was placed in energy regeneration subsystem. The fundamental elements of the storage subsystem are two $58 \mathrm{~F}$ Maxwell ultra-capacitors used for the storage of electric energy. By using two capacitors, one can obtain larger amount of regenerated energy and larger efficiency of the storage process because the resultant capacitance is larger while the charging rate is rather small. In the proposed circuit, the first capacitor $C_{1}$ is charged immediately, and the second $C_{2}$ is charged indirectly by $\mathrm{DC} / \mathrm{DC}$ converter. For the assumed excitation, the maximum relative velocity of the engine core with respect to the engine coil equals approximately $0.1 \mathrm{~m} / \mathrm{s}$. In this case the maximum voltage across the linear motor coil is less than $2.5 \mathrm{~V}$.

\section{Experimental studies}

The laboratory workstation allows for testing the harvesting system in the range of frequency up to $50 \mathrm{~Hz}$. The measurements were performed for the following parameters of the workstation: $m_{1}=3.8 \mathrm{~kg}, m_{2}=2.9 \mathrm{~kg}$, $k_{1}=10500 \mathrm{~N} / \mathrm{m}, k_{2}=6567 \mathrm{~N} / \mathrm{m}, b_{1}=30 \mathrm{Ns} / \mathrm{m}$, $b_{2}=5 \mathrm{Ns} / \mathrm{m}$.

Before starting measurements relating to the energy accumulating system, motion of the mechanical subsystem was considered in the case when the circuit of the motor was closed and thus the energy accumulating system was made inactive. The whole of energy transferred from mechanical subsystem into electrical energy was turned into heat in resistor. Vibrations of both masses were

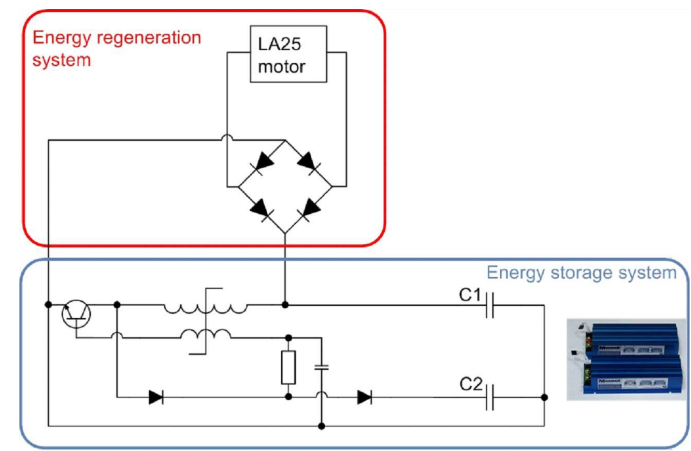

Fig. 8. Diagram of the energy regeneration system and energy storage system.

reduced. The support plate was subjected to vertical displacement $z(t)$. Sinusoidal excitation with frequency increasing from $f_{1}=0.2 \mathrm{~Hz}$ to $f_{2}=15 \mathrm{~Hz}$ at constant angular acceleration $\varepsilon=0.01 \mathrm{~Hz} / \mathrm{s}$ was assumed. The angular acceleration was so small, that the system could perform almost steady-state motion for all frequencies from the range $\left(f_{1}, f_{2}\right)$.

Using the registered values of displacements $x_{1}(t), x_{2}(t)$ and $z(t)$, the frequency response curves were calculated for both upper and lower mass. They are shown in Fig. 9.

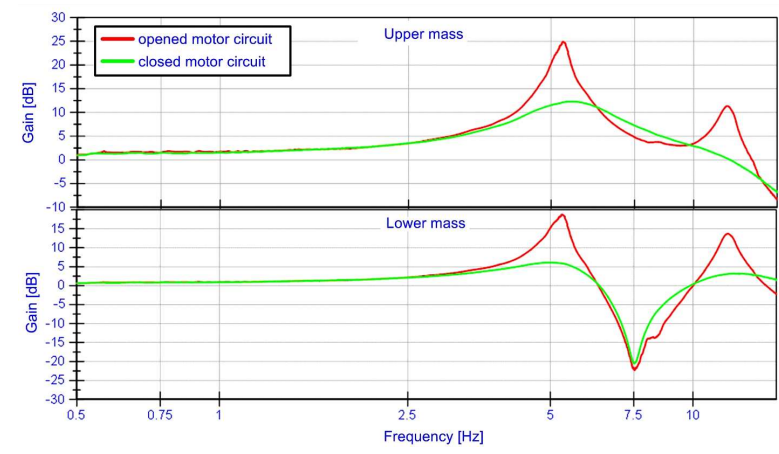

Fig. 9. Frequency response curves for upper and lower mass.

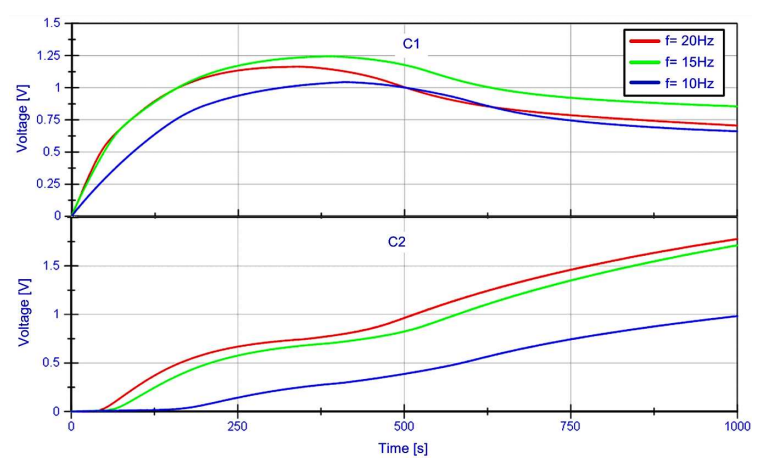

Fig. 10. Voltages across capacitors. 


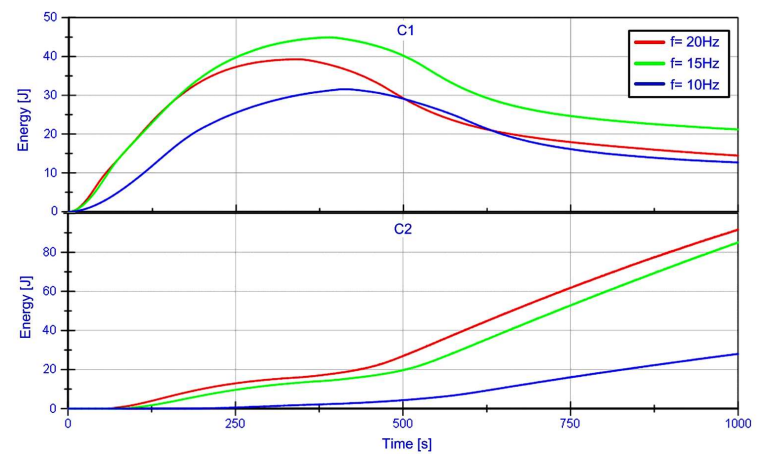

Fig. 11. Energies stored in capacitors.

In the next part of laboratory investigation, the energy accumulating system was active. The sinusoidal excitation with constant frequency $(10,15$, and $20 \mathrm{~Hz}$ ) and amplitude $A=1 \mathrm{~mm}$ was assumed. The scope of measurements was extended in order to investigate signals associated with the energy storage system. First of all, the current in the charging circuit and voltages across capacitors were measured. On the basis of the registered voltages, corresponding energies stored in capacitors were calculated. Results obtained for the three assumed frequencies are shown in Fig. 10 and Fig. 11. Additionally, as in the first part of the laboratory investigation, displacements $x_{1}(t), x_{2}(t)$, and $z(t)$ were measured.

Two stages can be distinguished in the charging process. In the first stage, from $t=0$ to $t=500 \mathrm{~s}$, charging of the first capacitor is the dominant phenomenon. The energy of the second capacitor increases very slowly. In the second stage, from $t=500$ to $t=1000 \mathrm{~s}$, the second capacitor is mostly charged. The first capacitor is slowly discharged. Its energy is partly transferred to the second capacitor. Application of two capacitors in the proposed system allows to decrease the charging rate and, as a consequence, to increase the energy storage efficiency.

\section{Summary}

In the paper, extraction of energy from vibrations of two-DOF mechanical system and the possibility of accumulation of the extracted energy in capacitors were analyzed. Using the simple model of a capacitor-charging circuit, efficiency of the energy storage process was investigated. The results of calculations were used in the design of energy storage system. With the use of dedicated laboratory workstation, a series of experiments have been performed. The experiments were oriented at determination of the storage system efficiency.

The main conclusions derived from calculations and measurements can be summarized as follows:

- Efficiency of the storage process depends mainly on the ratio of the source time parameter to the circuit time constant. For any time, large value of source time parameter ensures better efficiency of the energy storage process. According to definition of the source time parameter, the larger is its value, the lower is the charging process rate.

- Application of large capacitors in the charging circuit increases the circuit time constant. This can make efficiency of the storage process unsatisfactory. In the energy storage system, proposed in the paper, the drop of efficiency was essentially limited.

- The proposed harvesting system can be connected with a vibration control system acting in the suspension of the upper mass. In this case, energy stored in capacitors could be supplied to the vibration control system. A self-powered vibration control system can be formed in this way.

\section{References}

[1] M. Lallort, E. Lefeuvre, C. Richard, D. Guyomar, Sensors and Actuators: A. Physical 143, 377 (2008).

[2] K. Nakano, Y. Suda, S. Nakadai, H. Tsunashima, T. Washizum, JSAE Review 20, 511 (1999).

[3] K. Nakano, Y. Suda, S. Nakadai, J. Sound Vibr. 260, 213 (2003).

[4] T. Paing, J. Shin, R. Zane, Z. Popovic, IEEE Trans. Power Electr. 23, 1494 (2008).

[5] US Patent No. 6501325B1, Low Voltage Supply Higher Efficiency Cross-Coupled High Voltage Charge Pumps. 2002.

[6] Z. Stević, M. Rajčić-Vujasinović, S. Bugarinović, A. Dekanski, Acta Physica Polonica A 117, 228 (2010).

[7] G. Szarka, B. Stark, S. Burrow, IEEE Trans. Power Electr. 27, 803 (2012).

[8] M. Fodor, R. Redfield, Vehicle System Dynamics 22, 1 (1993).

[9] J. Snamina, J. Kowal, P. Orkisz, Acta Physica Polonica A 123, 1118 (2013). 